\title{
Computational benchmarking for ultrafast electron dynamics: wavefunction methods vs density functional theory
}

\author{
Micael J. T. Oliveira, ${ }^{*, \dagger, \ddagger}$ Benoit Mignolet, $₫$ Tomasz Kus, ${ }^{\Uparrow}$ \\ Theodoros A. Papadopoulos, ${ }^{\S}$ Françoise Remacle, $₫$ and Matthieu J. Verstraete ${ }^{\dagger, \ddagger}$ \\ Department of Physics, University of Liège, B-4000, Liège, Belgium, European Theoretical \\ Spectroscopy Facility http://www.etsf.eu, Department of Chemistry, University of Liège, \\ B-4000, Liège, Belgium, and Department of Natural Sciences, University of Chester, \\ Thornton Science Park, CH2 4NU, Chester, U.K. \\ E-mail: mjt.oliveira@ulg.ac.be
}

\begin{abstract}
Attosecond electron dynamics in small and medium size molecules, induced by an ultrashort strong optical pulse, is studied computationally for a frozen nuclear geometry. The importance of exchange and correlation effects on the non-equilibrium electron dynamics induced by the interaction of the molecule with the strong optical pulse is analyzed by comparing the solution of the time-dependent Schrödinger equation based on the correlated field-free stationary electronic states computed with the Equation-OfMotion Coupled Cluster Singles and Doubles (EOM-CCSD) and the Complete Active
\end{abstract}

\footnotetext{
${ }^{*}$ To whom correspondence should be addressed

${ }^{\dagger}$ Department of Physics, University of Liège, B-4000, Liège, Belgium

${ }_{\ddagger}^{\ddagger}$ European Theoretical Spectroscopy Facility http://www.etsf.eu

`Department of Chemistry, University of Liège, B-4000, Liège, Belgium

${ }^{\S}$ Department of Natural Sciences, University of Chester, Thornton Science Park, CH2 4NU, Chester, U.K.
} 
Space Multi-Configurational Self-Consistent Field (CAS-SCF) methodologies on one hand, and various functionals in real-time Time-Dependent Density Functional Theory (TD-DFT) on the other. We aim to evaluate the performance of the latter approach, which is very widely used for non linear absorption processes and whose computational cost has a more favorable scaling with the system size.

We focus on $\mathrm{LiH}$ as a toy model for a non-trivial molecule, and show that our conclusions carry over to larger molecules, exemplified by $\mathrm{ABCU}\left(\mathrm{C}_{10} \mathrm{H}_{19} \mathrm{~N}\right)$. The molecules are probed with IR and UV pulses whose intensities are not strong enough to significantly ionize the system. By comparing the evolution of the time dependent field-free electronic dipole moment, as well as its Fourier power spectrum, we show that TDDFT performs qualitatively well in most cases. Contrary to previous studies, we find almost no changes in the TD-DFT excitation energies when excited states are populated. Transition between states of different symmetries are induced using pulses polarized in different directions. We observe that the performance of TD-DFT does not depend on the symmetry of the states involved in the transition.

\section{Introduction}

The interaction of ultrashort and strong optical pulses with matter has been the focus of intense activity for the past two decades. Such pulses provide a direct analytic tool to probe the electronic states, and to prepare a non-equilibrium state of a molecule. ${ }^{1,2}$ Tailored pulses can generate a non-equilibrium electron density made of the coherent superposition of specific stationary electronic states, which could potentially steer chemical reactions, via isomerization, bond breaking, and reorganization. These processes occur in a time scale of the order of picoseconds or more, but their outcome is in principle controlled by the initial non-equilibrium (electronic) conditions. Potential applications abound, most prominently to catalysis, chemical reactivity, and biology. ${ }^{3}$

Experimental facilities dedicated to photo-physics and photo-chemistry have taken great 


\section{Page 3 of 36}

strides beyond the previous (femtosecond) state of the art. ${ }^{4}$ Several powerful techniques give access to optical pulses at the attoesecond time scale, such as X-Ray Free Electron Lasers, ${ }^{5}$ and High Harmonic Generation (HHG). ${ }^{6}$ Single attosecond pulses have been isolated ${ }^{7}$ from HHG pulse trains, giving access to detailed attosecond probe or pump pulses, though the combination of both in an atto pump-probe experiment remains a challenge.

Electron dynamics can be probed on the attosecond time scale, while typical ionic motion resides in the picosecond regime, as the fastest movements last dozens of femtoseconds. An advantage of attosecond photo-chemistry is that the preparation of electronic states should be less perturbed by or entangled with the movement of the nuclei, as is the case with femto and longer pulses. One can probe the resulting motion as a function of the time delay between pump and probe pulses before the onset of significant nuclear motion: longer time delays would give access to the vibronic dynamics. The corollary is that short attosecond pulses have a large energy bandwidth, which can result in strong entanglement between electronic states. This can also be exploited to tailor a specific non equilibrium electronic density by adjusting the characteristics of the short strong optical pulse, such as carrier wave length, polarization, duration, and carrier envelope phase.

A spectrum of different theories addresses the modelling of the electronic structure of stationary states for frozen nuclei, within the Born-Oppenheimer approximation, going from full configuration interaction (CI), which is in principle exact but scales exponentially with the number of electrons $N$, to coupled cluster $(\operatorname{CCSD}(\mathrm{T})),{ }^{8,9}$ which scales as $N^{5}$, and complete active space multiconfiguration self consistent field (CAS-SCF), ${ }^{10-13}$ which limits the effective number of electrons and active orbitals, to mean-field density functional theory (DFT), ${ }^{14,15}$ which usually scales as $N^{3}$. This list of theories is not exhaustive by any means; they are given in decreasing order of both accuracy and computational cost. Only few of these methods can be used to compute the electronic or nuclear dynamics in molecules, due to their computational cost. Even for small molecules, one usually focuses on a restricted number of nuclear degrees of freedom taking part in the reaction, and a small number of 
electronic states.

Simulating attosecond electron dynamics in molecules has received growing interest in the past few years, focusing mainly on diatomic $\left(\mathrm{H}_{2}, \mathrm{~N}_{2}, \mathrm{CO}, \mathrm{LiH}\right)$ and triatomic $\left(\mathrm{CO}_{2}, \mathrm{LiCN}\right)$ molecules, for which experimental data is available, the electronic structure is relatively simple, and highly accurate quantum chemical methods can be implemented. There are three main ways of solving the time-dependent Schrodinger equation. The first is to propagate orbitals, as it is done in real time TD-DFT, ${ }^{16}$ TD-CAS, $,{ }^{17-20} \mathrm{TD}-\mathrm{CI},{ }^{21}$ and R-matrix scattering theories. ${ }^{22}$ Another route consists in computing a basis of field-free electronic states, with a correlated method such as CI, ${ }^{23-26}$ coupled cluster, ${ }^{27,28}$ multi-reference SCF, ${ }^{29}$ or linear response TD-DFT, ${ }^{28,30}$ and then solving the time-dependent Schrödinger equation including the coupling to the electric field to all orders in the basis of field-free electronic states. It is therefore possible to obtain the time dependent populations of the field-free electronic states, as well as the dipole moment during the dynamics. Real-time propagation methods like TD-DFT only provide the time-dependent dipole moment, which is an observable of the density, unlike the populations. The populations can be extracted by comparing intensities with a linear response spectrum or projecting on ground state orbitals, but in this work we will mainly focus on the comparison of the time-dependent dipole moments. Finally, the explicit resolution of the Schrödinger equation on a grid is another method of choice, but essentially possible only for $\mathrm{H}_{2}$ and $\mathrm{H}_{2}^{+} \cdot{ }^{31-34}$

Real-time TD-DFT ${ }^{16}$ has the strong advantage of good system-size scaling, which allows for the calculation of ion as well as electron dynamics. Real-time propagation of the equations makes TD-DFT a simple first order differential equation, which scales remarkably well as $\mathrm{N}^{2}$. TD-DFT is thus a good compromise, and often the only choice for large systems. The central issue in the application of TD-DFT is that exchange and correlation (xc) effects are not well controlled or systematically improvable. One important feature of the xc potential is that it is a functional of the time-dependent density and has a non-local dependency on time. Approximate functionals are usually made adiabatic, that is, they depend on 


\section{Page 5 of 36}

the instantaneous density only. In any case, this means that the TD-DFT Hamiltonian includes an extra time-dependency in the xc potential, which in turn implies that, when an approximate xc functional is used, the excitation energies of the system might not be constant in time, and can change as the populations of the excited states change. ${ }^{35}$ Therefore, the TD-DFT excitation energies of a given system out of equilibrium may change in time, even in the absence of an external perturbation.

Although not as abundant as for ground-state properties, several benchmarks of xc functionals can be found in the literature for excited-state properties (see ref. 36 for a comprehensive review). Most of these benchmarks focus on the performance of TD-DFT to predict excitation energies and, to a lesser extent, transition dipole moments when the initial state is the ground-state. However, because of aforementioned spurious variation in time of the excitation energies, this information is not enough to infer the accuracy of TD-DFT to describe the electron dynamics in attosecond pulses. Several TD-DFT benchmarks of electron dynamics driven by optical pulses used simple model systems for which exact solutions of the time-dependent Schrödinger equation are available. ${ }^{37-42}$ These studies showed that approximate xc functionals very often fail to even qualitatively reproduce important features of the exact results, such as resonant Rabi oscillations, charge-transfer dynamics, or the dynamical step structures of the xc potential. Nevertheless, conclusions about the behavior of TD-DFT for model systems are not always straightforward to carry over to more realistic systems, so more specific benchmarks on small and medium-size molecules are essential to assess the performance of approximate xc functionals in TD-DFT simulations of ultrafast electron dynamics. In this vein, Raghunathan and Nest compared the attosecond electron dynamics in TD-CI Singles+Doubles with different flavors of TD-DFT, for the molecules $\mathrm{Li}_{2} \mathrm{C}_{2}$ and LiCN. ${ }^{25,43}$ They found mixed results and claimed that the best agreement between TD-DFT and TD-CISD was obtained when not too much energy was pumped to the molecules, and for transitions where the dipole moments of the ground and final states had similar directions and magnitudes. They also noted the time-dependence of the excitation energies, and 
showed it could seriously hinder the usage of TD-DFT in coherent control theory. ${ }^{25,44}$ Sonk and Schlegel ${ }^{28}$ have studied butadiene, comparing TD-CI populations starting from a number of different linear response TD-DFT approximations, and using RPA and EOM-CCSD as references. They conclude on the importance of long range correction, and that several LC-GGA functionals give excellent results with respect to CCSD and experiment, both for excitation position and for the residual state population after a pulse. Semilocal and hybrid DFT functionals provide too low excitation energies and IPs, and are populated much too strongly by an IR pulse.

In the following, we attempt to validate and expand previous TD-DFT benchmarks by investigating the role of exchange and correlation in attosecond electron dynamics induced by ultrashort optical pulses in a small molecule $(\mathrm{LiH})$ with well separated potential energy surfaces (PES), and on a larger molecule $\left(\mathrm{ABCU}\right.$ or $\left.\mathrm{C}_{10} \mathrm{H}_{19} \mathrm{~N}\right)$ with a principal $\mathrm{C}_{3}$ axis. We compare the time-dependent solution of the Schrödinger equation in a basis of multideterminant field-free states (EOM-CCSD and CAS-SCF), with different functionals for real-time TD-DFT. CAS-SCF ${ }^{10-13}$ and EOM-CCSD ${ }^{8,9,45-47}$ were chosen as representatives of two widely used complementary methods based on multi-determinant wave functions for computing transition energies and wave functions of excited electronic states. CAS-SCF is variational for both the determinants and LCAO coefficients on a restricted active space while EOM-CCSD uses a truncated expansion of the many electron wave function on determinants. For the 4 electron molecule $\mathrm{LiH}$, both methodologies are expected to be close to the FCI limit. These methods are thus among the most accurate, yet computationally affordable for the benchmarked systems. We focus on the electron dipole moment, as the simplest observable which is common to all theories, and examine its response to short and strong optical pulses with IR or UV frequencies. ABCU is beyond the reach of full coupled cluster methodology, and is treated only within the complete active space (CAS-SCF) quantum chemistry method. Because of their $\mathrm{C}_{3}$ symmetries, the low lying $\mathrm{A}$ and $\mathrm{E}$ excited states of ABCU present strong similarities with the $\Sigma$ and $\Pi$ states of the $\mathrm{C}_{\infty V} \mathrm{LiH}$ molecule. 


\section{Methodology}

\subsection{Optical aborption cross-section}

For each molecule under study we start by calculating the optical absorption cross-section, which gives the probability of absorption as a function of photon frequency. This quantity can be obtained in a straightforward way with the CAS-SCF and EOM-CCSD methods, as those methods allow to calculated the excitation energies of the system and the corresponding transition dipole moments, which in turn are trivially related to the oscillator strengths. In the case of TD-DFT, the excitation energies and oscillator strengths can also be obtained directly using linear-response theory through the well known Casida method. Here, however, we have used a different method, by calculating the optical absorption from real-time TDDFT. ${ }^{48,49}$ In this method the ground-state of the system is perturbed by a dipolar electric field that acts at $t=0$ and excites equally all the frequencies of the system:

$$
\delta v(\mathbf{r}, t)=-\mathbf{E} \cdot \mathbf{r} \delta(t)
$$

We then solve the time-dependent Kohn-Sham equations to obtain the time-dependent density, $n(\mathbf{r}, t)$, and compute the time-dependent dipole moment:

$$
\boldsymbol{\mu}(t)=\int \mathrm{d} \mathbf{r} \mathbf{r} n(\mathbf{r}, t)
$$

From the induced dipole moment, $\delta \boldsymbol{\mu}(\omega)$, we obtain the components of the dynamical polarizability tensor:

$$
\alpha_{i j}(\omega)=\frac{\delta \mu_{i}(\omega)}{E_{j}},
$$

which are trivially related to the optical absorption cross-section tensor:

$$
\sigma_{i j}(\omega)=\frac{4 \pi \omega}{c} \mathfrak{I m}\left[\alpha_{i j}(\omega)\right]
$$


Note that in the last two equations all the quantities were moved from time domain to the more convenient frequency domain.

\section{$2.2 \quad$ Ultrashort optical pulse}

Based on the results for the optical absorption cross-section, we choose a set of ultrashort optical pulses to probe a representative range of excited states of the system. All the pulses are taken to have a gaussian envelope:

$$
\mathbf{E}(t)=\mathbf{E} e^{\frac{-\left(t-t_{0}\right)^{2}}{2 \sigma^{2}}} \cos (\omega t+\phi)
$$

where $t_{0}$ and $\sigma$ are the center and width of the envelope, respectively, $\omega$ is the carrier frequency, $\phi$ is the carrier envelope phase (CEP), and $\mathbf{E}$ defines both the field strength and polarization direction. In some cases the pulse is so short that it is a one cycle pulse. In such cases the electric field is defined including a correction to ensure that no DC or low frequency components are present. ${ }^{50}$

With attosecond pulses, where the field is strong and the frequency bandwidth quite wide, it is essential to go beyond the linear and perturbative regime and perform a real-time propagation of the systems interacting with the external field. After the end of the pulse, the system is propagated further in time and we keep track of the time-dependent dipole moment, $\boldsymbol{\mu}(t)$. To obtain the relevant spectra of electronic transitions, a Fourier transformation (FT) is then applied to the time-dependent dipole moment vector components after the pulse window. The modulus squared of the FT gives the power spectrum of the dipole moment. Note that, because the FT excludes the pulse window, what is computed is the power spectrum of the field-free dipole moment and it contains information about the excitation energies and transition dipole moments between states present in the wave packet after the pulse. We found that total time-propagations of $T \geq 48$ fs for $\mathrm{LiH}$ and of $T \geq 20$ fs for ABCU were sufficient to obtain a good resolution in the peak positions and relative intensities of the 


\section{Page 9 of 36}

dipole power spectrum. All the dipole moments in the first 20 fs are shown in the Supporting Information.

\subsection{TD-DFT}

We use DFT and TD-DFT as implemented in the OCTOPUS code, ${ }^{48,51}$ which has an emphasis on solving the time-dependent Kohn-Sham equation in real-time. In OCTOPUS, the main quantities are discretized in a regular rectangular real-space grid. Therefore, it is possible to improve in a systematic way the convergence with respect to the grid parameters: the spacing between the grid points and the size of the simulation box. We found that grid spacings of 0.22 a.u. ( $\mathrm{LiH}$ ) and 0.32 a.u. (ABCU) and simulation boxes constructed from a union of spheres of radius 16 a.u. ( $\mathrm{LiH})$ and 18 a.u. (ABCU) centered at the nuclei are necessary to converge the position of the main spectral peaks within $0.1 \mathrm{eV}$ in all our calculations. In the case of $\mathrm{LiH}$, in order to explicitly include all the electrons in the calculations we use Hartwigsen-Goedecker-Hutter pseudopotentials ${ }^{52}$ with all the electrons included in the valence space. For ABCU, we use the standard norm-conserving pseudopotentials distributed with the OCTOPUS package. In the case of the time-dependent calculations, we found that time steps of 0.02 a.u. ( $\mathrm{LiH})$ and 0.05 a.u. (ABCU) were small enough to ensure the stability of the time-dependent propagation, which in turn is carried out using the approximated enforced time-reversal symmetry propagator with the exponential of the Hamiltonian being numerically calculated using a Taylor expansion. ${ }^{53}$

The laser field is included in the Kohn-Sham Hamiltonian in the dipole approximation. In such a regime, using real time propagation allows us to extract electromagnetic properties that go beyond the commonly used linear response Casida approach.

Several hundreds of functionals have been proposed for DFT, ${ }^{54}$ from which we have selected qualitatively different representatives. The TD-DFT baseline is the local density approximation (LDA), which we compare here with the hybrid B3LYP functional ${ }^{55,56}$ and the average-density self-interaction correction (ADSIC), ${ }^{57}$ whose improved asymptotic tail 
for the potential should be important for charge transfers and excitations. Because the computational cost of applying the Fock operator in real-space is considerably more than the use of local and semi-local DFT functionals, we limit the use of B3LYP to LiH. All functionals will be considered adiabatic, i.e. $v_{x c}(t)=v_{x c}[n(t)]$ where $n(t)$ is the instantaneous density. It has been shown that the functional should have memory and spatial non-local dependencies, ${ }^{58,59}$ but these are very complex and little is known about their properties.

\subsection{Time-dependent propagation of multi-determinant wave func- tions}

For comparison, the electronic dynamics is computed by solving the time-dependent Schrödinger equation (TDSE) numerically in a basis of the multi-determinant field-free electronic states of the molecule. ${ }^{19,60}$ These states diagonalize the stationary electronic Hamiltonian. The full electronic Hamiltonian used in the TDSE includes the coupling of the molecule with the time-dependent electric field of the pulse in the dipole approximation. This coupling is therefore included to all orders, as in the TD-DFT methodology. The electronic structure of the field-free electronic states (energy and multi-determinant wave functions) and the transition dipole matrix elements can be obtained with any electronic structure method able to compute a band of excited states. Here the computation of the field free electronic states was done using the MOLPRO ${ }^{61}$ implementation of EOM-CCSD ${ }^{8,9,45-47}$ for LiH, and CAS$\mathrm{SCF}^{10-13}$ for both $\mathrm{LiH}$ and ABCU. The CAS-SCF approach has been extensively used in our previous work on $\mathrm{LiH}^{62,63}$ and $\mathrm{ABCU} .{ }^{29} \mathrm{CAS}-\mathrm{SCF}$ is recognized to be able to describe well static correlation effects and charge transfer excited states, including doubly excited states, provided the active space (number of electron and number of orbitals) is chosen with care. ${ }^{10-13,64}$ For describing coherent electronic dynamics in a band of excited states, one needs to use CAS-SCF average, which leads to systematic errors in the transition frequencies since the energy is minimized for an average wave function over the band of electronic states. EOM-CCSD is expected to better describe dynamic electron correlation ${ }^{9,45-47}$ and is is im- 


\section{Page 11 of 36}

plemented to get all the excited states that converge top the IP, by adding diffuse function on each atom center, see also Ref. ${ }^{27,28}$ EOM-CCSD is expected to give accurate transitions frequencies for singly excited states, which is the case for the LiH molecule investigated here. By the diagrammatic construction of the method, we expect properties related to the wave functions of higher excited states are also accurately described by EOM-CCSD. Since the transition dipoles depend on the quality of the wave functions, one of our purposes was to compare the values of the transition dipoles in the two methodologies. It indeed turns out that for LiH, CAS-SCF transitions dipoles are systematically larger than those computed in EOM-CCSD. There are not yet experimental results for highly excited electronic states and, therefore, no systematic way to judge how accurate the average CAS-SCF and EOM-CCSD transition frequencies and dipoles are for these states.

For the LiH molecule, a band of 40 electronic states $(15 \Sigma, 20 \Pi$, and $5 \Delta$ ) has been computed in CAS-SCF average including all the 4 electrons in the active space and 29 orbitals $(13 \sigma, 7 \pi$, and $2 \delta)$, in the $6-311++\mathrm{g}(2 \mathrm{df}, 2 \mathrm{p})$ basis set. ${ }^{65}$ We also computed a band of 63 states $(23 \Sigma, 40 \Pi)$ in EOM-CCSD methodology as implemented in MOLPRO using the aug-cc-pVQZ basis set, further augmented by the following diffuse functions (exponents in parenthesis): on Li, $3 \mathrm{~s}$ functions (0.00318, 0.001 and 0.0001), $2 \mathrm{p}$ functions $(0.003253$ and 0.001), $1 \mathrm{~d}$ function (0.0133), $1 \mathrm{f}$ function (0.0226), and $1 \mathrm{~g}$ function (0.05); on $\mathrm{H}, 5 \mathrm{~s}$ functions $(0.012815,0.0064,0.0032,0.001$, and 0.0001), $2 \mathrm{p}$ functions (0.0424 and 0.01), $1 \mathrm{~d}$ function (0.095), and $1 \mathrm{f}$ function (0.18). These very diffuse basis functions were added to describe correctly the states close the ionization potential.

For the ABCU molecule, the electronic structure of a band of 20 states has been computed in CAS-SCF average in an active space of 8 electrons and 13 orbitals, and with the $6-31++\mathrm{G}(\mathrm{d}, \mathrm{p})$ basis set. ${ }^{29}$ 


\section{Results}

\section{$3.1 \quad \mathrm{LiH}$}

The ground and excited states are computed in all approaches for nuclei fixed at the experimental geometry according to Ref. 66 . The accessible electronic states will be of either $\Sigma$ or $\Pi$ symmetry with respect to the main axis. The computed energies of the $\Delta$ states are all above the IP.

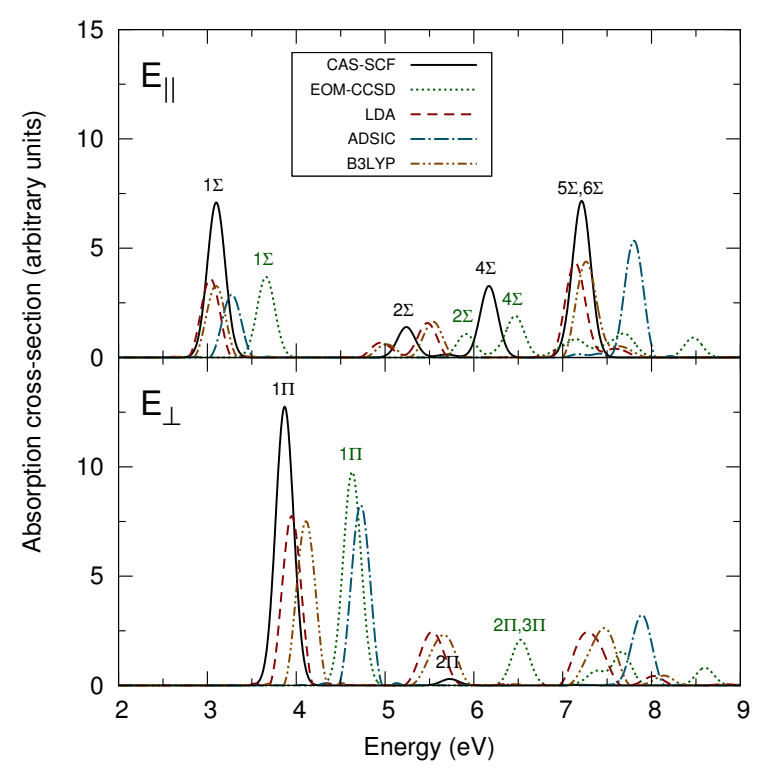

Figure 1: Optical absorption cross-section of $\mathrm{LiH}$ as a function of energy for light polarized parallel (top panel) and perpendicular (bottom panel) to the main molecular axis.

Table 1: Ground-state dipole moment $\mu_{G S}$ (a.u.), excitation energies $\Delta \mathrm{E}_{G S-1 \Sigma}$ and $\Delta \mathrm{E}_{G S-1 \Pi}$ (eV), and transition dipole moments $\mu_{G S-1 \Sigma}$ and $\mu_{G S-1 \Pi}$ (a.u.) of $\mathrm{LiH}$ obtained with different methods.

\begin{tabular}{lccccc}
\hline Method & $\mu_{G S}$ & $\Delta \mathrm{E}_{G S-1 \Sigma}$ & $\mu_{G S-1 \Sigma}$ & $\Delta \mathrm{E}_{G S-1 \Pi}$ & $\mu_{G S-1 \Pi}$ \\
\hline CAS-SCF & 2.10 & 3.11 & -1.39 & 3.87 & -1.67 \\
EOM-CCSD & 2.31 & 3.66 & -0.93 & 4.63 & -1.34 \\
TD-LDA & 2.21 & 3.05 & -1.00 & 3.96 & -1.29 \\
TD-ADSIC & 1.80 & 3.29 & -0.86 & 4.74 & -1.22 \\
TD-B3LYP & 2.23 & 3.09 & -0.95 & 4.11 & -1.25 \\
\hline
\end{tabular}

In Fig. 1 we plot the absorption cross-section of $\mathrm{LiH}$ up to $9 \mathrm{eV}$ obtained with different 


\section{Page 13 of 36}

methods for light polarized parallel, $\mathbf{E}^{\|}$, and perpendicularly, $\mathbf{E}^{\perp}$, to the main molecular axis. Within the dipole approximation for the field, standard optical selection rule implies that only excited states with $\Sigma$ symmetry are accessed with the parallel polarization, while only excited states with $\Pi$ symmetry are accessed with the perpendicular polarization. The excitation energies and the corresponding transition dipole moments of the $1 \Sigma$ and $1 \Pi$ excited states are also given in Table 1 . These values are directly related to the absorption crosssection spectra, as the position of the peaks is determined by the energy of the excited states and the oscillator strengths are proportional to the square of the corresponding transition dipole moments. For the lowest excited $\Sigma$ and $\Pi$ states, we observe some considerable differences in the obtained results, especially between CAS-SCF and EOM-CCSD. Overall, CAS-SCF gives larger transition dipole moments, while the EOM-CCSD excitation energies are systematically higher by $0.5-0.75 \mathrm{eV}$. The TD-DFT excitation energies fall between those calculated via CAS-SCF and EOM-CCSD: TD-LDA excitation energies are very close to the CAS-SCF ones, while the TD-ADSIC ones are higher in energy and, for the case of the 1П state, almost on top of the corresponding EOM-CCSD excitation energy. TD-B3LYP excitation energies are slightly higher in energy than the TD-LDA ones. This is the expected behavior, as B3LYP includes a small fraction of Hartree-Fock, which gives excitation energies considerably larger than the usual (semi-)local DFT functionals. The TD-DFT transition dipole moments agree reasonably well with those obtained via EOM-CCSD, although they seem to be underestimated by TD-ADSIC. In Table 1 the field-free ground-state dipole moment is also given. The CCSD ground-state dipole is in excellent agreement with the experimental value of 2.31 a.u., ${ }^{67}$ while all the other methods seem to underestimate it, with ADSIC having the largest deviation of $22 \%$ with respect to experiment.

For the higher lying excited states, care must be taken when analyzing the results obtained with TD-LDA and TD-B3LYP. Indeed, the exchange potential of LDA has an incorrect asymptotic behavior (the potential decays exponentially instead of exhibiting the correct $-1 / r$ behavior). As a consequence, the eigenvalue of the highest-occupied Kohn-Sham or- 
bital will be severely overestimated, which means that the electron occupying the HOMO is less bound and thus easier to photo-ionize. This, in theory, would not be a problem if the continuum of unbound Kohn-Sham states was accurately described. Unfortunately this is not the case, as that would require using a complete basis set to numerically represent the orbitals, which, in the case of a real-space grid, implies a simulation box of infinite size. Therefore, in our TD-DFT simulations, the unbound Kohn-Sham states are not accurately described. Because the LDA ionization potential (IP) obtained from Koopman's theorem is around $4.4 \mathrm{eV}$, some excited states above $5 \mathrm{eV}$ have an unbound Kohn-Sham orbital populated and are thus poorly described and strongly depend on the details of the simulation box. This strong dependence on the simulation box can be used to identify such states and we observe that the position and intensity of the TD-LDA peaks found at around 5, 5.5, and $7 \mathrm{eV}$ change continuously with the size of the simulation box. Therefore, the qualitative agreement found between TD-LDA and CAS-SCF in Fig. 1 for the corresponding $\Sigma$ states is fortuitous. Although B3LYP includes a fraction of the Hartree-Fock exchange, which has the correct asymptotics, this fraction is not enough to significantly improve the value of the IP and the situation for the excited states above $5 \mathrm{eV}$ is the same as for LDA. Note that, strictly speaking, this problem is not restricted to TD-LDA or to TD-DFT, but it is less severe for methods based on Hartree-Fock, which has the correct asymptotic behavior, or for xc functionals that have better asymptotics, like ADSIC. Indeed, we obtain a value of $9.4 \mathrm{eV}$ for Koopman's IP calculated with ADSIC. For this reason we will only discuss the absorption cross-section obtained with TD-ADSIC, EOM-CCSD, and CAS-SCF. For both light polarizations we observe large qualitative and quantitative discrepancies between the results obtained by all the methods for the states lying above $5 \mathrm{eV}$. For the $2 \Sigma$ and $3 \Sigma$ excited states, the discrepancies between CAS-SCF and EOM-CCSD seem to be only quantitative, with the latter yielding higher excitation energies and lower transition dipole moments. In the case of TD-ADSIC, these two excited states seem to be either absent or at much higher energies. 


\section{Page 15 of 36}

Table 2: Carrier frequencies, $\omega$, and intensities, $E^{\|}$and $E^{\perp}$, of the laser pulses applied to LiH.

\begin{tabular}{cccccc}
\hline Pulse & $\omega$ (a.u.) & $\omega(\mathrm{eV})$ & $\omega(\mathrm{nm})$ & $E^{\|}($a.u. $)$ & $E^{\perp}($ a.u. $)$ \\
\hline IR & 0.0570 & 1.55 & 800 & 0.01 & 0.02 \\
UV1a & 0.1150 & 3.13 & 396 & 0.005 & 0.01 \\
UV1b & 0.1345 & 3.66 & 339 & 0.005 & 0.0025 \\
UV2a & 0.1480 & 4.03 & 308 & 0.01 & 0.005 \\
UV2b & 0.1709 & 4.65 & 267 & 0.005 & 0.0025 \\
\hline
\end{tabular}

Based on the absorption spectra of Fig. 1, we choose five different excitation pulses. All the pulses are defined using Eq. 5 with $\sigma=60$ a.u. (ca. $1.5 \mathrm{fs}$ ) and $\phi=0$. The intensities and carrier frequencies are summarized in Table 2. The UV frequencies are chosen to be roughly resonant with the CAS-SCF and EOM-CCSD excitation energies of the first two excited states $(1 \Sigma$ and $1 \Pi)$. The intensities are chosen such that the exciting laser power is not so large as to remove an important fraction of an electron from the molecule. This is done by choosing a pulse intensity small enough to minimize the populations of the highly excited states obtained with CAS-SCF and EOM-CCSD during the pulse. This is crucial, because these states have large photo-ionization widths and once reached ionize almost instantaneously. ${ }^{63}$ In particular, in our real-space TD-DFT calculations, when the excitation pulse input power is sufficiently large, the electrons spread throughout the simulation box and can be reflected by the boundaries, as there is no way for them to leave the box.

Below we compare the different field frequencies and polarizations to show the effect of resonance, multi photon absorption, and symmetry on the power spectrum of the dipole moment. According to the selection rules, for a field polarized parallel to the main molecular axis, $E^{\|}$, the only allowed transitions are between $\Sigma$ states. Therefore, the $E^{\|}$pulses will build a superposition of $\Sigma$ states only. These states will be visible in the power spectrum of the parallel dipole moment component, $\mu_{\|}$, while the power spectrum of the perpendicular component of the dipole moment, $\mu_{\perp}$, will be zero by symmetry. Therefore, only the power spectrum of $\mu_{\|}$will be plotted for $E^{\|}$. In the case of a field polarized perpendicularly to the 
main molecular axis, $E^{\perp}$, the only allowed transitions from the ground-state are to $\Pi$ states. Once $\Pi$ states are populated, further transitions are possible, either to $\Sigma$ or to $\Delta$ states. All these transitions between states with different symmetries will be visible in the power spectrum of $\mu_{\perp}$. The variation in time of $\mu_{\|}$will be made of a superposition of beatings between states with the same symmetry. Therefore, the corresponding power spectrum will only contain peaks at frequencies that correspond to energy differences between populated $\Sigma$ states or between populated $\Pi$ states.

(a)

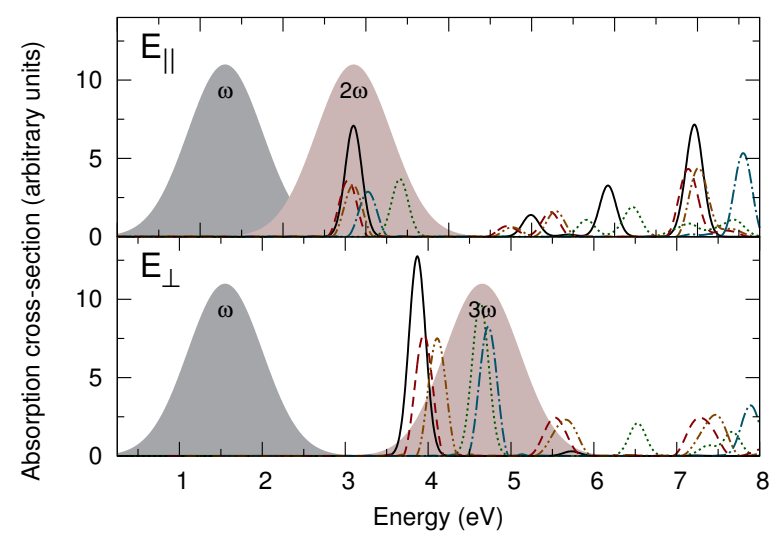

(c)

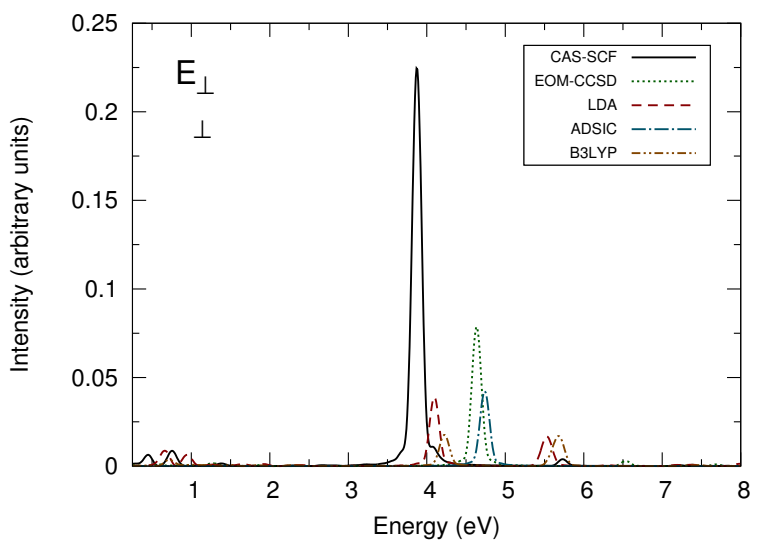

(b)

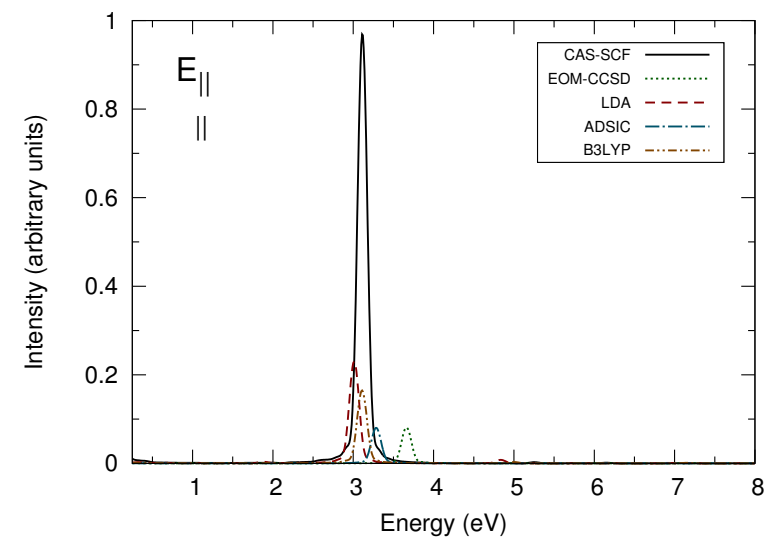

(d)

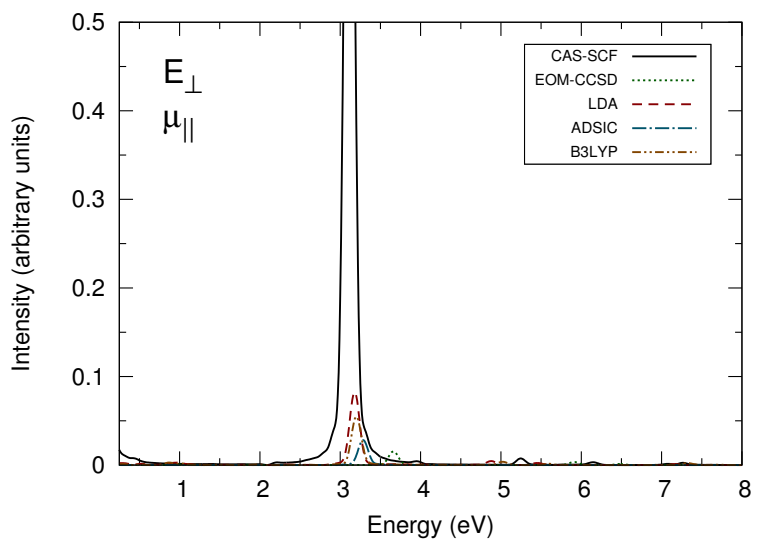

Figure 2: (a) LiH optical absorption spectra. The frequency components of the IR field as well as the same pulse with double or triple of the original frequency are shown as filled curves. (b) Parallel induced dipole moment power spectra for the IR pulse with the field parallel to the main molecular axis. (c)-(d) Parallel and perpendicular induced dipole moment power spectra for the IR pulse with the field perpendicular to the main molecular axis. 


\section{Page 17 of 36}

The first situation we consider is for low frequency IR pulses. As explained above, when the electric field of the pulse is parallel to the molecular axis, only $\Sigma$ states can be accessed, either by one or multi-photons transitions. From Fig. 2(a) we observe that the CAS-SCF, EOM-CCSD, TD-LDA and TD-B3LYP $1 \Sigma$ states are two photon resonant with the pulse and the corresponding peaks are visible at around $3 \mathrm{eV}$ in the power spectrum of the dipole parallel to the molecular axis shown in Fig. 2(b). The difference in the peak heights given by these methods is related to the differences in the corresponding transition dipole moments. The same state calculated by EOM-CCSD and TD-ADSIC is found to be higher in energy and thus slightly out of resonance, which explains not only the position of the peaks in the power spectrum, but also their reduced intensities. For the pulse polarized perpendicularly to the molecular axis, the $\Pi$ states are accessible by one or three photon transitions. From the absorption spectra it is visible that the TD-ADSIC and EOM-CCSD 1П states are three photon resonant, with the CAS-SCF, TD-LDA, and TD-B3LYP 1П states close enough to be also accessible. As in the case of the parallel pulse, the different relative intensities between the methods seen in Fig. 2(c) can be explained by how close the excited state is to resonance with the pulse and by the differences in the transition dipole moments. For the perpendicular pulse, although a direct transition from the ground-state to a $\Sigma$ state is dipole forbidden, these states are still accessible through a two step process: photon absorption to get to a $\Pi$ state and then re-emission or new absorption to get to a $\Sigma$ state. The small peaks below $1 \mathrm{eV}$ confirm that these processes do occur, as their position corresponds to the energy difference between the $1 \Pi$ state and the $1 \Sigma$ and $2 \Sigma$ states. This can be further confirmed by looking at the power spectrum of the dipole moment along the molecular axis in Fig. 2(d), which tells us which $\Sigma$ states are populated. It is observed that $\Sigma$ states are populated for all methods on the same energy window, which is not apparent in the power spectrum of Fig. 2(c). Finally, comparing the excitation energies given by the position of the dipole power peaks with the excitation energies obtained from linear response (see Table S1 from the Supporting Information), no significant shifts, that is, shifts larger than $0.1 \mathrm{eV}$, 
are observed for the parallel pulse. For the perpendicular pulse shifts of around $0.1 \mathrm{eV}$ are observed in the excitation energies of the $1 \Sigma$ and $1 \Pi$ states obtained with TD-LDA and TD-B3LYP . As mentioned in the Introduction, this type of behavior can be traced back to the use of an approximated exchange-correlation functional ${ }^{35}$ and has been observed in the literature before. ${ }^{25,44}$

(a)

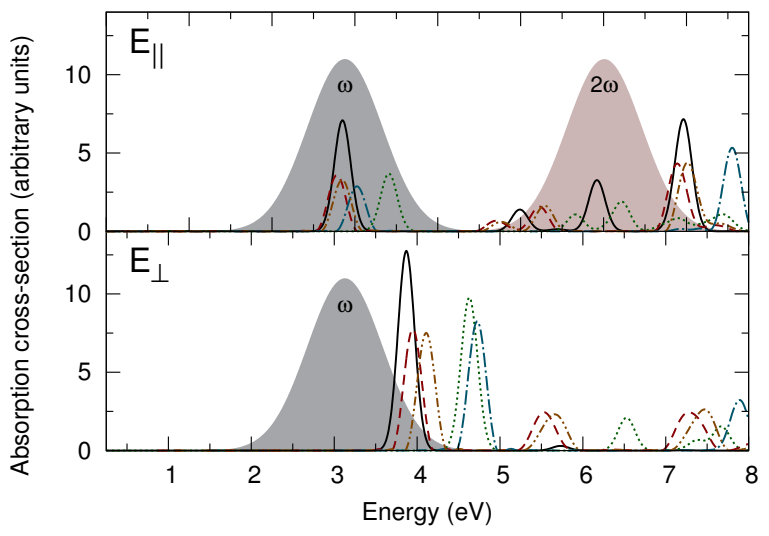

(c)

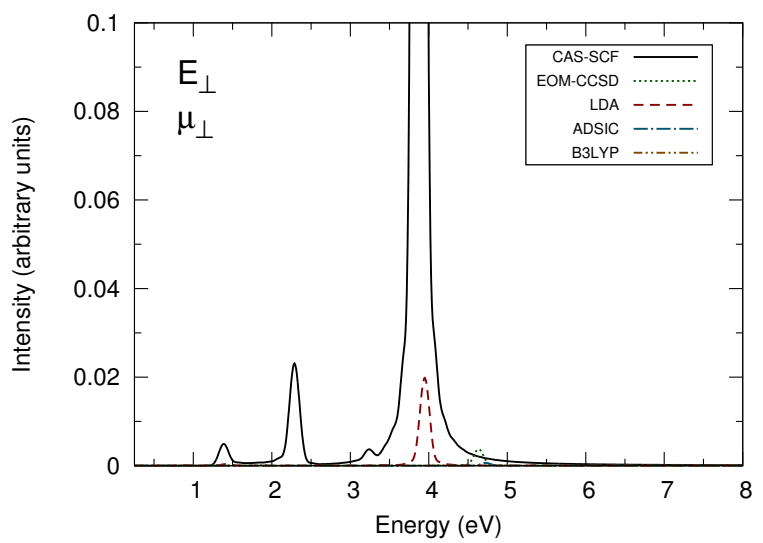

(b)

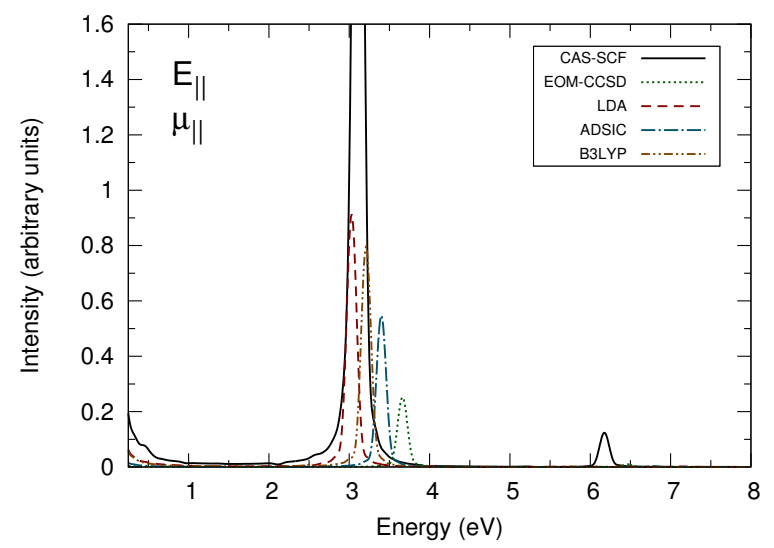

(d)

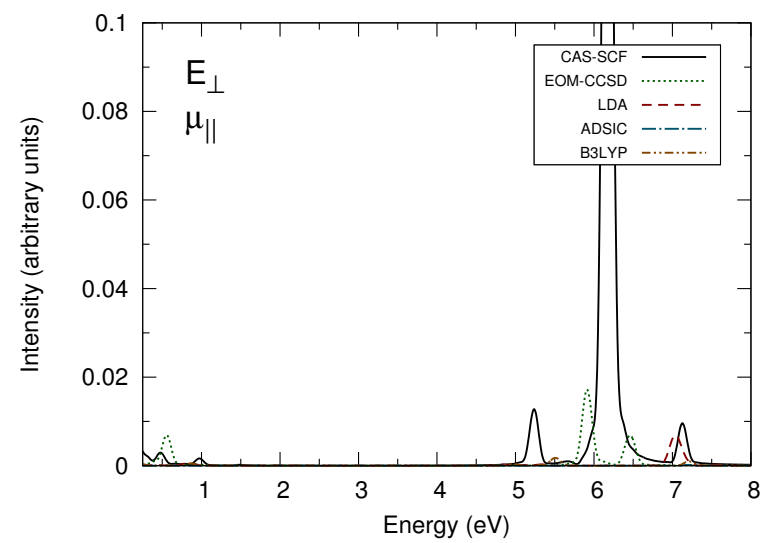

Figure 3: (a) LiH optical absorption spectra. The frequency components of the UV1a field as well as the same pulse with double the original frequency are shown as filled curves. (b) Parallel induced dipole moment power spectra for the UV1a pulse with the field parallel to the main molecular axis. (c)-(d) Parallel and perpendicular dipole moment power spectra for the UV1a pulse with the field perpendicular to the main molecular axis.

We then considered the response to a field resonant with the first CAS-SCF $\Sigma$ state. For 


\section{Page 19 of 36}

the pulse parallel to the molecular axis, we see from Fig. 3(a) that the $1 \Sigma$ state is directly accessible and the pulse is broad enough so that this state is populated by all methods, despite the differences in excitation energies. We observe well-defined peaks in Fig. 3(b) corresponding to the transition from the ground-state to the $1 \Sigma$ state in all cases. As in the case of the IR pulse, the relative intensities of the peaks correspond to the differences in the transition dipole moments and, in the case of TD-ADSIC and EOM-CCSD, to the energy difference between excited state and the resonant pulse frequency. In the case of CAS-SCF, a second transition is also visible at around $6.2 \mathrm{eV}$, which corresponds to a two photon transition to a higher $\Sigma$ state. When the field is perpendicular to the molecular axis, the pulse is still broad enough to populate the $1 \Pi$ state, as can be seen in Fig. 3(c), even if a considerable population of that state is only achieved for CAS-SCF. Because the energy differences between the CAS-SCF $1 \Pi$ and the $3 \Sigma$ states is close to the carrier frequency, a distinct peak can be observed at around $2.3 \mathrm{eV}$ for the CAS-SCF spectra, which corresponds to a transition from the $1 \Pi$ state to the $3 \Sigma$ state. Similarly, two smaller peaks can be seen at around 1.4 and $3.2 \mathrm{eV}$, which correspond to transitions from the $1 \Pi$ state to the $2 \Sigma$ and $4 \Sigma$ states respectively. It is possible to confirm that these $\Sigma$ states are indeed populated, as the corresponding peaks can be observed at around 5 and $7 \mathrm{eV}$ in the power spectra of the parallel dipole shown in Fig. 3(d). The same figure shows that the $2 \Sigma$ and $3 \Sigma$ EOM-CCSD states are also populated, although this is not visible in Fig. 3(c). In this case, we note small shifts of $0.1 \mathrm{eV}$ in the excitation energies of the $1 \Sigma$ state for the parallel pulse with TD-ADSIC and TD-B3LYP.

As can be seen in Fig. 1 and in Tab. 1, there is a difference of more than $0.5 \mathrm{eV}$ in the excitation energy of the $1 \Sigma$ state as calculated with CAS-SCF and with EOM-CCSD. Since this energy difference has important consequences in the relative intensities of the peaks of the dipole power spectrum when the pulse is resonant with the CAS-SCF state, we now apply a pulse that is resonant with the corresponding EOM-CCSD $1 \Sigma$ state to see the effect on the spectra. For the pulse parallel to the molecular axis, the relative intensities of the main 
(a)

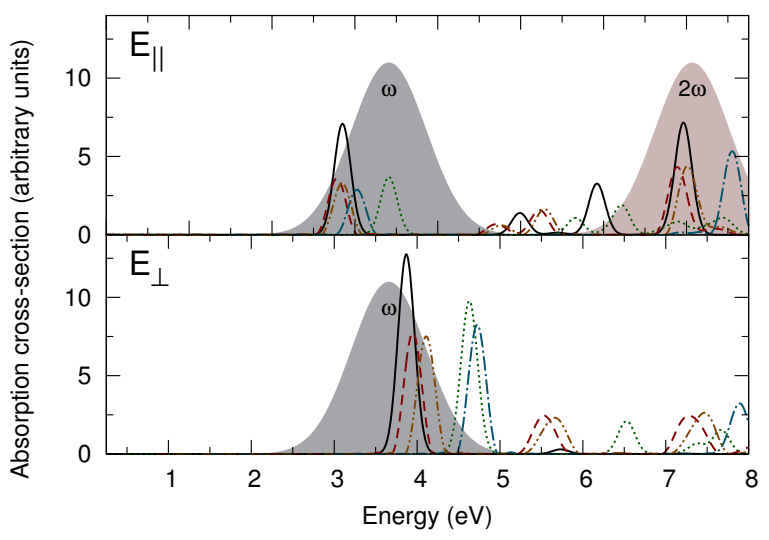

(c)

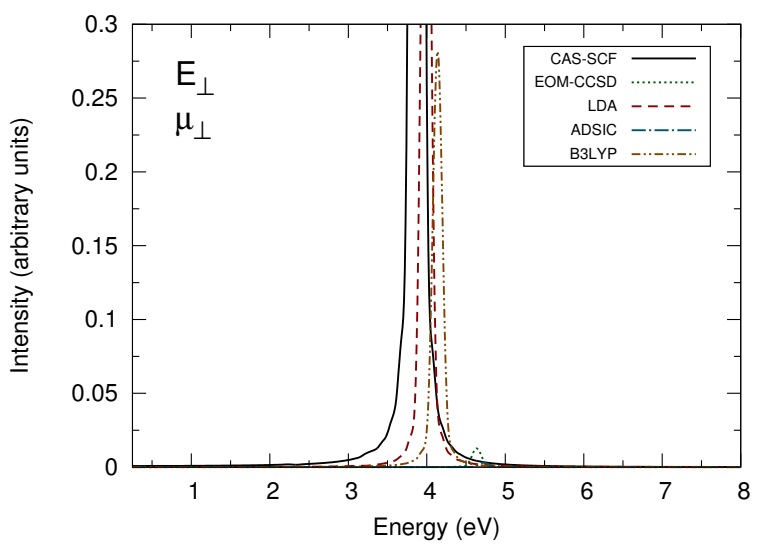

(b)

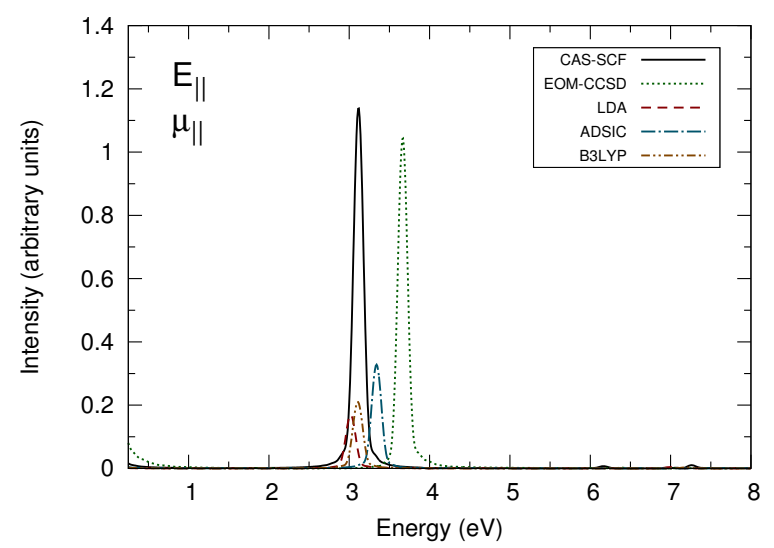

(d)

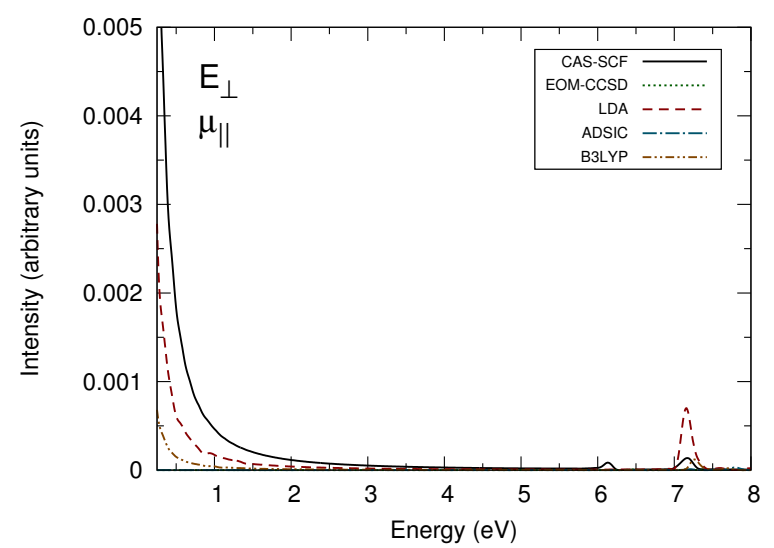

Figure 4: (a) LiH optical absorption spectra. The frequency components of the UV1b field as well as the same pulse with double the original frequency are shown as filled curves. (b) Parallel induced dipole moment power spectra for the UV1b pulse with the field parallel to the main molecular axis. (c)-(d) Parallel and perperdicular dipole moment power spectra for the UV1a pulse with the field perpendicular to the main molecular axis.

peaks of Fig. 4(b) are now inverted with respect to the CAS-SCF resonant case (Fig. 3(b)), which is the expected result considering that it is now the EOM-CCSD $1 \Sigma$ state that is resonant with the pulse frequency. In Figs. 4(c) and 4(d) the power spectrum of the parallel and perpendicular components of the dipole moment are plotted in the case of the pulse polarized perpendicularly to the molecular axis. In this case the carrier frequency of the field is less than $0.6 \mathrm{eV}$ from resonance with the CAS-SCF, TD-LDA, and TD-B3LYP 1П 


\section{Page 21 of 36}

states, which explains why these states are considerably populated. No signatures of $\Pi$ to $\Sigma$ transitions are observed in this case, due to the field intensity being four times smaller than for the previous UV1a pulse. For this pulse, no significant shifts of the excitation energies are seen in the plots.

(a)

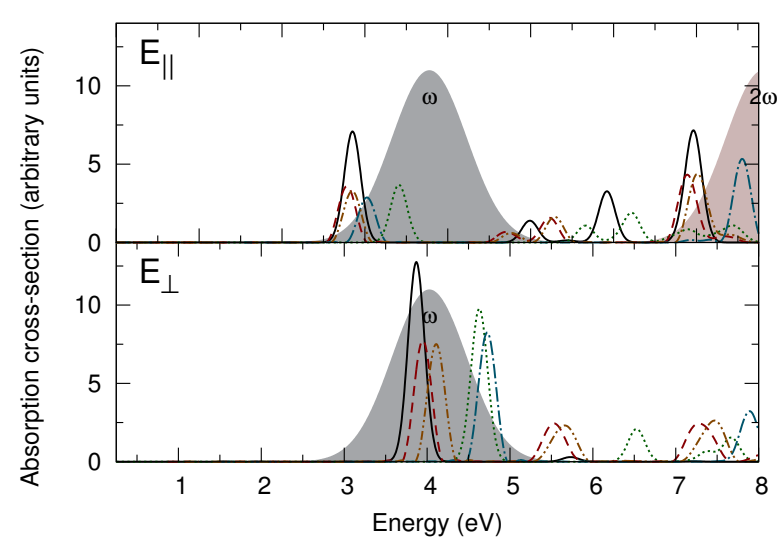

(c)

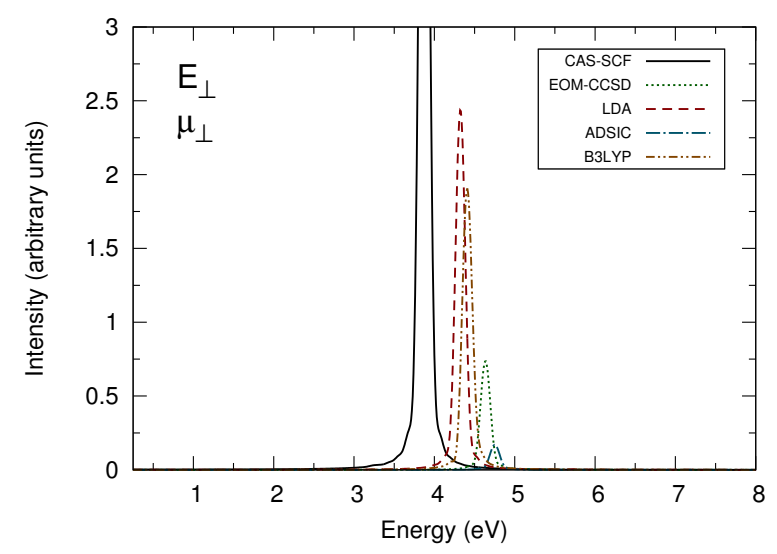

(b)

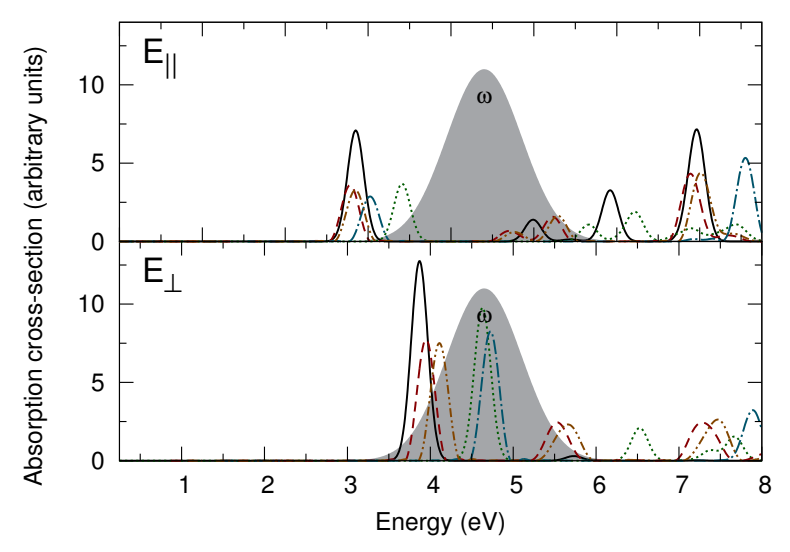

(d)

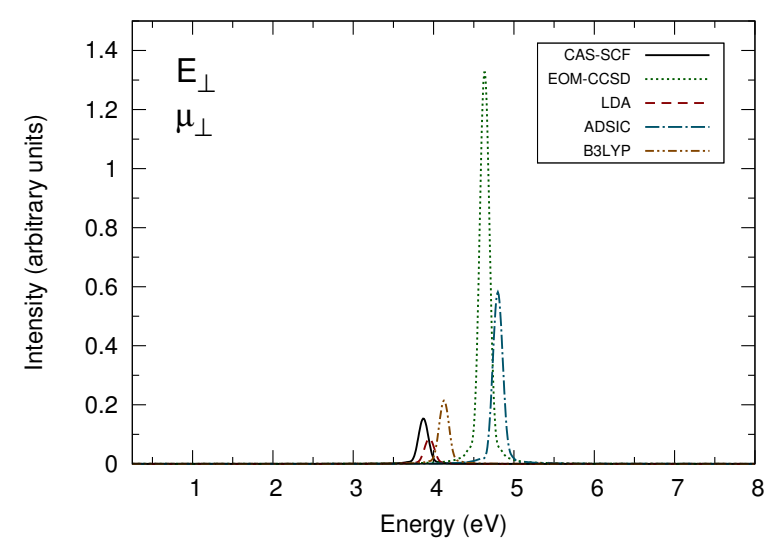

Figure 5: (a) LiH optical absorption spectra. The frequency components of the UV1a field are shown as filled curves. (b) Perpendicular induced dipole moment power spectra for the UV2a pulse with the field perpendicular to the main molecular axis. (c) LiH optical absorption spectra. The frequency components of the UV1b field are shown as filled curves. (d) Perperdicular dipole moment power spectra for the UV2b pulse with the field perpendicular to the main molecular axis.

The final two cases considered are for UV fields resonant with the CAS-SCF and EOMCCSD 1П states (Fig. 5). For a pulse parallel to the molecular axis (see Supporting Informa- 
tion), the results can be deduced from the optical absorption spectra and, in this respect, are very similar to the previous UV1a and UV1b cases. For the pulse polarized perpendicularly to the molecular axis, the $1 \Pi$ states are populated by one photon transitions and the corresponding transition is readily identified in Figs. 5(c) and 5(d). The relative intensities of the peaks obtained with the different methods are again explained by the differences in the corresponding transition dipole moments and by how close to resonance are the excitation energies. Nevertheless, we note one important difference with respect to the previous cases: in Fig. 5(c) we observe that the excitation energies of the TD-LDA and TD-B3LYP 1П states for the UV2a pulse are shifted towards higher energies by more than $0.3 \mathrm{eV}$ with respect to the corresponding excitation energies found in the optical absorption. From all the pulses considered, this is the case where the largest shifts are found.

\section{$3.2 \quad \mathrm{ABCU}$}



Figure 6: Equilibrium geometry of neutral ABCU obtained from CAS-SCF. ${ }^{29}$ The molecule belongs to the $\mathrm{C}_{3}$ symmetry group and the main molecular axis passes by the $\mathrm{N}$ and $\mathrm{C}_{4}$ atoms.

All quantum chemical calculations are performed at the equilibrium geometry of the neutral, optimized in CAS-SCF with 8 active electron, 13 orbitals, and the $6-31++\mathrm{G}(\mathrm{d}, \mathrm{p})$ 


\section{Page 23 of 36}

basis set. As discussed in the Introduction, the electronic states of $\mathrm{ABCU}$ in the $\mathrm{C}_{3}$ geometry belong either to the A symmetry (analogous to the $\Sigma$ symmetry of a diatomic molecule) or to the doubly degenerate E symmetry that is analogous to the $\Pi$ states. Moreover, due to the asymmetry between the $\mathrm{N}$ (top) and C4 (bottom) part of the cage (see Fig. 6), ABCU behaves as a hetero-nuclear diatomic molecule with a size of $3 \AA$. ABCU is a rigid cage with a high fragmentation energy. ${ }^{68}$

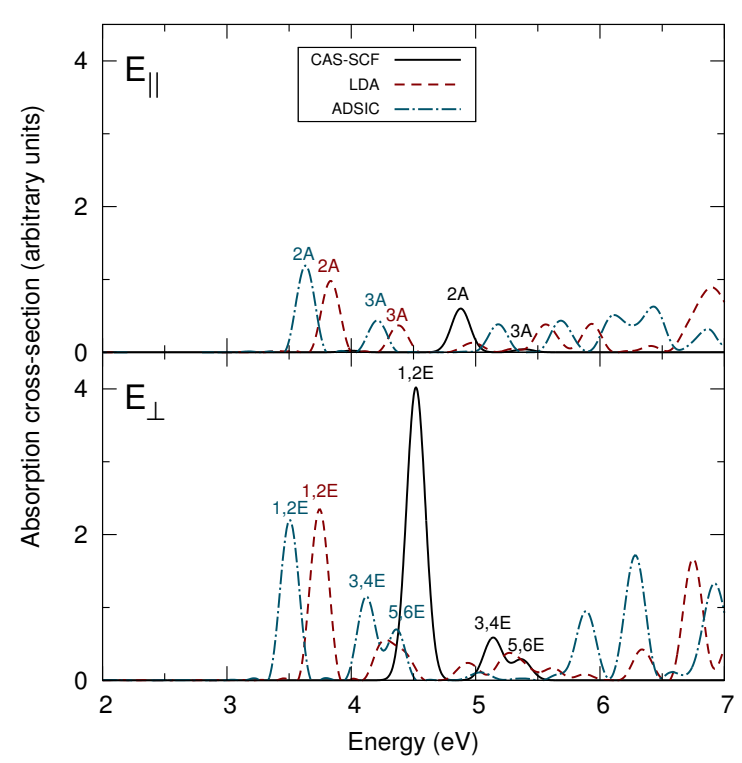

Figure 7: Optical absorption cross-section of ABCU as a function of energy for light polarized parallel (top panel) and perpendicular (bottom panel) to the main molecular axis.

The ABCU optical absorption cross section computed using CAS-SCF, TD-LDA, and TD-ADSIC is shown in Fig. 7. The excitation energies and transition dipole moments of the first nine excited states are also given in Table 3. Like in the case of LiH, the incorrect asymptotics of the LDA potential leads to a poor description of some of the excited states, namely the ones above $4.5 \mathrm{eV}$. In the case of ADSIC this value is $6.4 \mathrm{eV}$. We observe that all the excitation energies are strongly underestimated by both TD-LDA and TD-ADSIC when compared to the CAS-SCF results. This underestimation appears to be systematic, with most of the TD-LDA excited states being around $0.8 \mathrm{eV}$ lower in energy than the corresponding CAS-SCF states, while this value is $1.0 \mathrm{eV}$ for TD-ADSIC. Nevertheless, in 
Table 3: Excitation energies (eV), and transition dipole moments (a.u.) of selected ABCU excited states (ES) obtained with different methods.

\begin{tabular}{|c|c|c|c|c|c|c|c|c|c|c|c|c|}
\hline \multirow[b]{3}{*}{ ES } & & & & \multicolumn{9}{|c|}{ Transition dipole moment (a.u.) } \\
\hline & \multicolumn{3}{|c|}{ Excitation Energy (eV) } & \multicolumn{3}{|c|}{ TD-LDA } & \multicolumn{3}{|c|}{ TD-ADSIC } & \multicolumn{3}{|c|}{ CAS-SCF } \\
\hline & TD-LDA & TD-ADSIC & CAS-SCF & $\mu_{x}$ & $\mu_{y}$ & $\mu_{z}$ & $\mu_{x}$ & $\mu_{y}$ & $\mu_{z}$ & $\mu_{x}$ & $\mu_{y}$ & $\mu_{z}$ \\
\hline $1 \mathrm{~A}$ & 3.46 & 3.22 & 4.00 & 0.00 & 0.00 & -0.02 & 0.00 & 0.00 & -0.01 & 0.00 & 0.00 & 0.06 \\
\hline $1 \mathrm{E}$ & 3.75 & 3.51 & 4.52 & -0.58 & 0.26 & 0.00 & -0.59 & 0.24 & 0.00 & 0.65 & 0.38 & 0.00 \\
\hline $2 \mathrm{E}$ & 3.75 & 3.51 & 4.52 & 0.26 & 0.58 & 0.00 & -0.24 & -0.59 & 0.00 & -0.39 & 0.65 & 0.00 \\
\hline $2 \mathrm{~A}$ & 3.84 & 3.64 & 4.88 & 0.00 & 0.00 & -0.39 & 0.00 & 0.00 & -0.44 & 0.00 & 0.00 & -0.28 \\
\hline $3 \mathrm{E}$ & 4.26 & 4.13 & 5.14 & 0.29 & 0.03 & 0.00 & 0.30 & -0.29 & 0.00 & -0.06 & 0.26 & 0.00 \\
\hline $4 \mathrm{E}$ & 4.26 & 4.13 & 5.14 & 0.03 & -0.29 & 0.00 & 0.29 & 0.31 & 0.00 & -0.26 & -0.07 & 0.00 \\
\hline $3 \mathrm{~A}$ & 4.39 & 4.22 & 5.39 & 0.00 & 0.00 & 0.23 & 0.00 & 0.00 & 0.25 & -0.13 & -0.13 & 0.00 \\
\hline $5 \mathrm{E}$ & 4.42 & 4.36 & 5.37 & 0.24 & 0.05 & 0.00 & 0.32 & 0.06 & 0.00 & 0.13 & -0.13 & 0.00 \\
\hline $6 \mathrm{E}$ & 4.42 & 4.36 & 5.37 & -0.05 & 0.24 & 0.00 & -0.07 & 0.32 & 0.00 & 0.00 & 0.00 & 0.07 \\
\hline
\end{tabular}

the case of the energy differences between excited states, there is a much lower deviation of around $0.1 \mathrm{eV}$ of the TD-DFT results with respect to the CAS-SCF ones. This implies that the TD-DFT spectra are roughly shifted by $0.8-1.0 \mathrm{eV}$ towards lower energies with respect to the CAS-SCF spectra. As for the peak intensities, they seem to be systematically overestimated with TD-LDA and TD-ADSIC, with the notable exception of the 1E and 2E excited states, which are strongly underestimated.

Table 4: Parameters of the optical pulses applied to ABCU.

\begin{tabular}{cccccccc}
\hline Pulse & $\omega_{C A S-S C F}(\mathrm{eV})$ & $\omega_{L D A}(\mathrm{eV})$ & $\omega_{A D S I C}(\mathrm{eV})$ & $\hat{\mathbf{E}}$ & $|\mathbf{E}|($ a.u. $)$ & $\sigma$ & $\phi$ \\
\hline $\mathrm{IR}^{+}$ & 1.52 & 1.25 & 1.17 & $\perp$ & 0.03 & 25 & 0 \\
$\mathrm{IR}^{-}$ & 1.52 & 1.25 & 1.17 & $\perp$ & 0.03 & 25 & $\pi$ \\
$\mathrm{UV}^{+}$ & 4.90 & 3.84 & 3.62 & $\|$ & 0.05 & 30 & 0 \\
$\mathrm{UV}^{-}$ & 4.90 & 3.84 & 3.62 & $\perp$ & 0.02 & 30 & $\pi$ \\
\hline
\end{tabular}

In this case we used four different pulses taken from Ref. 29. In that work the authors used CAS-SCF, and the frequencies of some of the pulses were chosen to be resonant with the excitation energy of the $2 \mathrm{~A}$ excited state $(4.9 \mathrm{eV})$, while some IR pulses were roughly threephoton resonant with the $1 \mathrm{E}$ and $2 \mathrm{E}$ excited states $(4.6 \mathrm{eV})$. Because of the large differences in the excitation energies given by TD-LDA and TD-ADSIC with respect to the CAS-SCF, we decided to perform TD-DFT calculations with UV pulses always resonant with the $2 \mathrm{~A}$ 


\section{Page 25 of 36}

excited state and the IR pulses always three photon resonant with the $1 \mathrm{E}$ and $2 \mathrm{E}$ excited states (for the given xc functional). All the pulse parameters are summarized in Table 4.

(a)

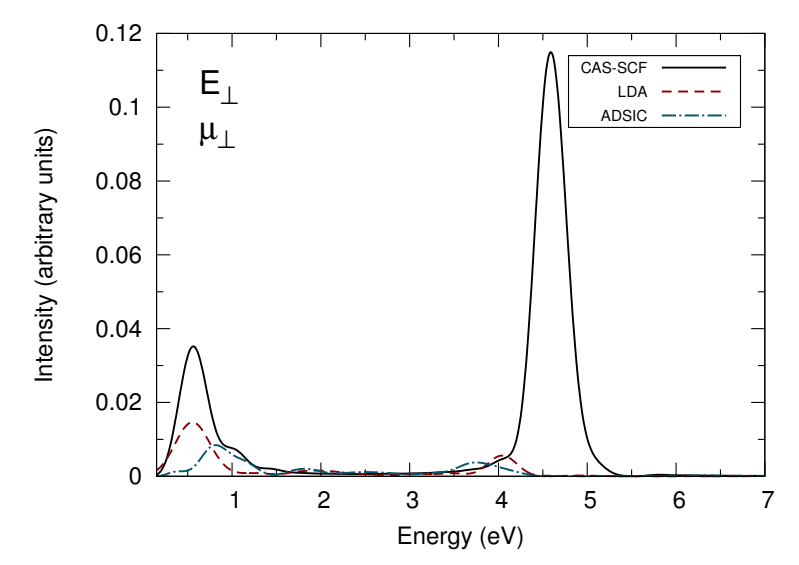

(b)

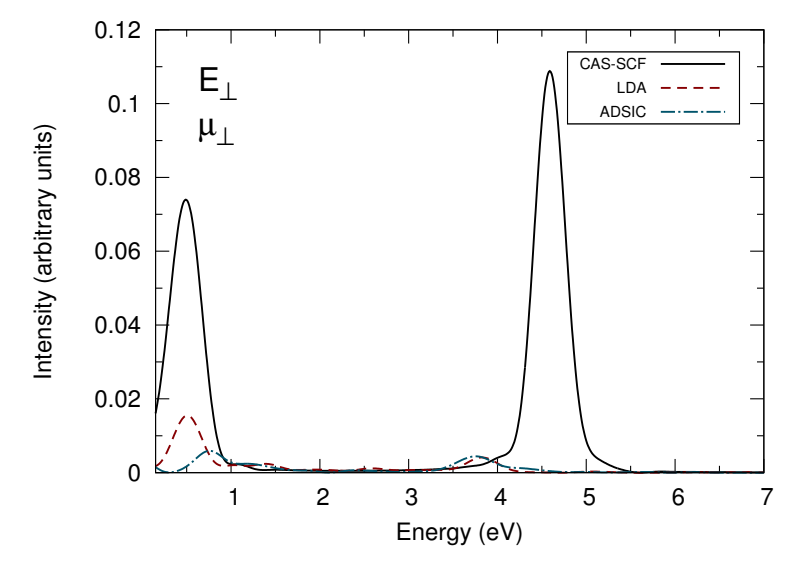

Figure 8: (a) Perpendicular induced dipole moment power spectra for the $\mathrm{IR}^{+}$pulse with the field perpendicular to the main molecular axis. (b) Perpendicular induced dipole moment power spectra for the $\mathrm{IR}^{-}$pulse with the field perpendicular to the main molecular axis.

The first case we consider is the excitation by two identical IR pulses with opposite carrier envelope phases. As shown in Ref. 29, the difference in the phases means that the density will be localized in different parts of the molecule during the pulses and so the transient dynamics will be different, which will lead to slightly different populations of the excited states. Because the pulses are polarized perpendicularly to the main molecular axis, the excited states of E symmetry can be accessed through a multi-photon transition. Once a E 
state is populated, further transitions to excited states with A symmetry become possible. In Fig. 8 we observe peaks between 3.5 and $4.5 \mathrm{eV}$ corresponding to the $1 \mathrm{E}$ and $2 \mathrm{E}$ excited states, which are three photon resonant with the pulse. Other peaks can be seen at lower energies, which correspond to transitions from the $1 \mathrm{E}$ and $2 \mathrm{E}$ states to excited states with A symmetry. No significant shifts are observed in the excitation energies obtained from TDDFT with respect to the ones in Fig. 7. On the other hand, large discrepancies are found for the relative intensities of the peaks between the different methods. These can be partially explained by the differences in the transition dipole moments predicted by the different methods. There are two noteworthy qualitative differences between the TD-DFT results and the CAS-SCF ones. First, the relative intensities of the transition from the ground-state to the $1 \mathrm{E}$ and $2 \mathrm{E}$ excited states with respect to the other transitions at lower energy seem to be inverted. This is because the transition dipole moment between the ground-state and the $1 \mathrm{E} / 2 \mathrm{E}$ states in TD-DFT is underestimated. Second, TD-DFT predicts smaller changes than CAS-SCF in the intensity of the transitions from the $1 \mathrm{E} / 2 \mathrm{E}$ states to the excited states with A symmetry when the CEP changes. As shown in Ref. 29, the opposite CEP gives rise to different transient dynamics and, therefore, different pairs of states are slightly populated during the pulse, which induces the change in the peaks near $1 \mathrm{eV}$. Because the transition dipole moments between the excited states are large, it is enough to have $0.1 \%$ of population in one highly excited state to observe the peak near $1 \mathrm{eV}$. This means that the observed differences between the TD-DFT and CAS-SCF results are not necessarily caused by large differences in the excited states populations. Comparing the excitation energies given by the position of the dipole power peaks with the excitation energies obtained from linear response (see Table S2 from the Supporting Information), the situation for ABCU seems to be somewhat worse than for $\mathrm{LiH}$ : shifts between 0.2 and $0.3 \mathrm{eV}$ are found for the excitation energies of the $1,2 \mathrm{E}$ states for the $\mathrm{IR}^{+}$pulse for both TD-LDA and TD-ADSIC, and for the $\mathrm{IR}^{-}$pulse for TD-ADSIC.

The second case considered is the response to two UV pulses resonant with the 2A state, 


\section{Page 27 of 36}

(a)

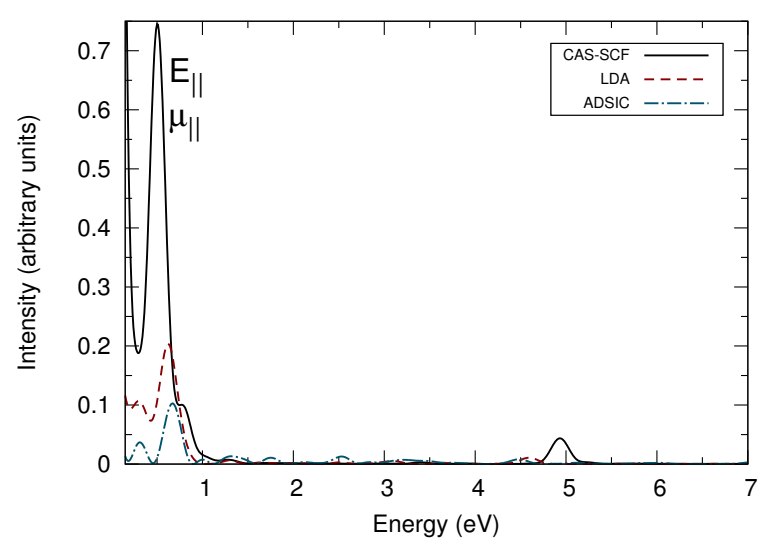

(b)

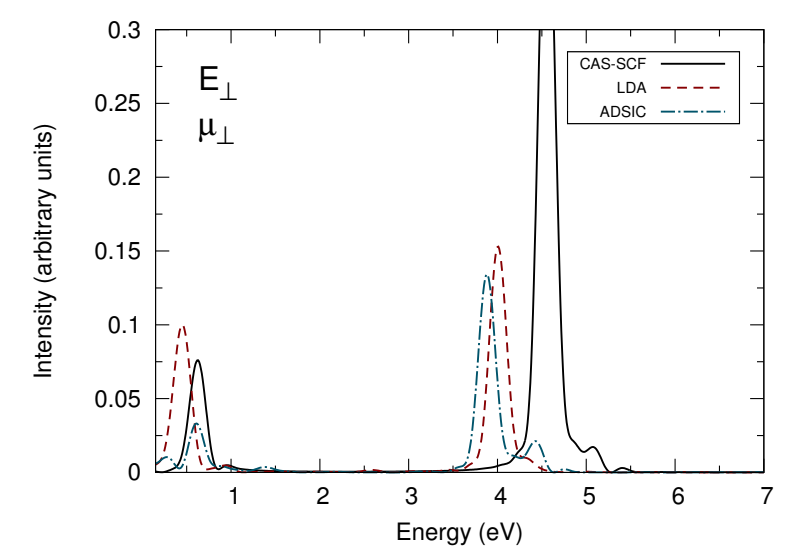

Figure 9: (a) Parallel induced dipole moment power spectra for the $\mathrm{UV}^{+}$pulse with the field parallel to the main molecular axis. (b) Perpendicular induced dipole moment power spectra for the $\mathrm{UV}^{-}$pulse with the field perpendicular to the main molecular axis.

but polarized along different directions, and with opposite carrier envelope phases. Their intensities are set by Ref. 29 with a criterion to limit the occupancy of autoionizing states. For the $\mathrm{UV}^{-}$pulse, the electric field is polarized perpendicularly to the main molecular axis and the allowed transitions are the same as for the previous IR pulses. In Fig. 9(b) we observe that the pulse considerably populates the excited states with E symmetry found between 3.5 and $5 \mathrm{eV}$. Further transitions occur from the $1 \mathrm{E}$ and $2 \mathrm{E}$ states to excited states with A symmetry, as can be seen in the lower part of the same spectrum. In all cases a good agreement is found between all methods. For the $\mathrm{UV}^{+}$pulse, the electric field is polarized 
parallel to the main molecular axis and the allowed transitions are to excited states with A symmetry. In Fig. 9(a), several small peaks can be observed between 3 and $5.5 \mathrm{eV}$, which correspond to transitions from the ground-state to A states. From these states further transitions are possible to other A states, either by further photon absorption or by emission. These transitions correspond to the peaks found below $3 \mathrm{eV}$. Overall the three methods give similar results, with CAS-SCF predicting higher intensities, as usual. The most significant difference is the higher number of excited states accessible in the TD-DFT cases, which results in a higher number of peaks in the corresponding spectra. Concerning the excitation energies of the 1,2E states, like for the previous IR case, shifts of around $0.3 \mathrm{eV}$ are observed for the $\mathrm{UV}^{-}$pulse for both TD-LDA and TD-ADSIC.

\section{Conclusions}

We have compared the performance of EOM-CCSD, CAS-SCF, and TD-DFT methods (LDA, ADSIC, and B3LYP) via the simulation of real-time ultrafast electron dynamics in molecules excited by an attosecond pulse for frozen nuclear geometries. To compare the aforementioned methods we chose the power spectrum of the field-free induced electronic dipole moment as a function of time, after real-time propagation. Two molecules were examined, namely LiH, a small but non trivial case for which EOM-CCSD is still computationally affordable, and ABCU, a medium sized cage molecule with sufficient symmetry to provide distinct characters for the excited states and to simplify the analysis of the field-free dipole power spectra. For both molecules, probing different components of the dipole moment with pulses polarized in different directions allowed us to characterize different types of transitions between the states.

We find that TD-DFT is able to qualitatively reproduce the results obtained with the two other methods. This happens for fields that are sufficiently strong to take the system beyond the linear response regime, provided that the intensities are not strong enough for 


\section{Page 29 of 36}

the system to ionize, as ionization is not considered in our framework. This latter condition should be carefully considered when selecting an xc functional and makes functionals with an incorrect asymptotic behavior less suited for practical applications. This confirms the conclusions of Ref. 28 obtained for IR pulses. Here we also investigate UV pulses and two polarization directions of the electric field. The addition of CAS to EOM-CCSD (and RPA in Ref. 28) to the direct time TD-DFT results gives quite a representative set of wave function methods. The better performance of ADSIC in the present work is similar to the long range corrected functionals, but does not contain adjustable parameters or imply the cost of a full HF exchange term in $\mathrm{V}_{x c}$.

For the exchange-correlation functionals used in this work and for pulse intensities considered, we found almost no changes in the TD-DFT excitation energies once the excited states of the system start to be populated. This is in contrast with previous findings ${ }^{25,44}$ and implies that TD-DFT calculations using simple adiabatic functionals can still be suited to study attosecond dynamics of small and medium sized molecules when none or little ionization occurs, despite the known limitations and pathologies of such functionals. Also in contrast with previous studies, ${ }^{25,43,44}$ we found that TD-DFT performed equally well when the initial and final states of a given transition had either similar or different dipole moments, that is, the qualitative and quantitative performance of the xc functionals was not related to the symmetries of the states that were part of the wave-packet. For both of the molecules under study, and for all of the pulses considered, the attosecond molecular response depends principally on the transition frequencies and on the transition dipoles between the states. Computing this response quantitatively and reliably with TD-DFT requires improving the xc functional to obtain more accurate excitation energies and transition dipole moments. 


\section{Acknowledgements}

The authors would like to acknowledge Mathias Nest, Marc Vrakking, Miguel Marques, Alberto Castro, Neepa Maitra, and Franck Lépine for fruitful discussions. This work was funded by the Belgian Fonds National de la Recherche Collective through project number 2.4545.12 "Control of attosecond dynamics: applications to molecular reactivity". BM and FR acknowledge support from the Fonds National de la Recherche Scientifique (Belgium). Computer time was provided on Zenobe by the Walloon Region under GA 1117545, and by PRACE on ARCHER (ThermoSpin and ACEID 3IP FP7 RI-312763).

\section{Supplemental Material}

Figures with all the induced dipole moments of $\mathrm{LiH}$ for the UV2a and UV2b pulses, and figures of $\mathrm{LiH}$ and $\mathrm{ABCU}$ induced dipoles moments as a function of time are available in the Supplemental Material. This information is available free of charge via the Internet at http://pubs.acs.org/.

\section{References}

(1) Remacle, F.; Levine, R. D. Proc. Nat. Acad. Sci. USA 2006, 103, 6793-6798.

(2) Kling, M. F.; Vrakking, M. J. J. Annu. Rev. Phys. Chem. 2008, 59, 463-492.

(3) Brinks, D.; Hildner, R.; van Dijk, E. M. H. P.; Stefani, F. D.; Nieder, J. B.; Hernando, J.; van Hulst, N. F. Chem. Soc. Rev. 2014, 43, 2476-2491.

(4) Baum, P.; Zewail, A. H. In Ultrafast Phenomena XVI; Corkum, P., Silvestri, S., Nelson, K. A., Riedle, E., Schoenlein, R. W., Eds.; Springer Series in Chemical Physics; Springer Berlin Heidelberg, 2009; Vol. 92; pp 155-157.

(5) McNeil, B. W. J.; Thompson, N. R. Nature Photon. 2010, 4, 814-821. 


\section{Page 31 of 36}

(6) Plaja, L.; Torres, R.; Zair, A. Attosecond Physics Attosecond Measurements and Control of Physical Systems; Springer Series in Optical Sciences; Springer: Heidelberg, 2013.

(7) Vrakking, M. J. J. Phys. Chem. Chem. Phys. 2014, 16, 2775-2789.

(8) Bartlett, R. J. In Theory and Applications of Computational Chemistry: The First Forty Years; Dykstra, C. E., Frenking, G., Kim, K. S., Scuseria, G. E., Eds.; Elsevier: Amsterdam, 2005; pp 1191-1221.

(9) Bartlett, R. J.; Musiał, M. Rev. Mod. Phys. 2007, 79, 291-352.

(10) Eade, R. H. A.; Robb, M. A. Chem. Phys. Lett. 1981, 83, 362-368.

(11) Jorgensen, P.; Olsen, J.; Yeager, D. L. J. Chem. Phys. 1981, 75, 5802-5815.

(12) Roos, B. O. In Advances in Chemical Physics: Ab Initio Methods in Quantum Chemistry Part 2; Lawley, K. P., Ed.; John Wiley \& Sons, Inc.: Hoboken, NJ, USA, 1987; Vol. 69; pp 399-445.

(13) Werner, H.-J. In Advances in Chemical Physics: Ab Initio Methods in Quantum Chemistry Part 2; Lawley, K. P., Ed.; John Wiley \& Sons, Inc.: Hoboken, NJ, USA, 1987; Vol. 69; pp 1-62.

(14) Hohenberg, P.; Kohn, W. Phys. Rev. 1964, 136, B864-B871.

(15) Kohn, W.; Sham, L. J. Phys. Rev. 1965, 140, A1133-A1138.

(16) Marques, M. A. L., Maitra, N. T., Nogueira, F. M. S., Gross, E. K. U., Rubio, A., Eds. Fundamentals of Time-Dependent Density Functional Theory; Lecture Notes in Physics; Springer Verlag: Berlin, 2012; Vol. 837.

(17) Sato, T.; Ishikawa, K. L. Phys. Rev. A 2013, 88, 023402.

(18) Miyagi, H.; Madsen, L. B. Phys. Rev. A 2013, 87, 062511. 
(19) Remacle, F.; Nest, M.; Levine, R. D. Phys. Rev. Lett. 2007, 99, 182902.

(20) Nest, M.; Remacle, F.; Levine, R. D. New J. Phys. 2008, 10, 025019.

(21) Rohringer, N.; Gordon, A.; Santra, R. Phys. Rev. A 2006, 74, 043420.

(22) Serbinenko, V.; Smirnova, O. J. Phys. B: At. Mol. Opt. Phys. 2013, 46, 171001.

(23) Krause, P.; Klamroth, T.; Saalfrank, P. J. Chem. Phys. 2005, 123, 074105.

(24) Sonk, J. A.; Schlegel, H. B. J. Phys. Chem. A 2012, 116, 7161-7168.

(25) Raghunathan, S.; Nest, M. J. Chem. Phys. 2012, 136, 064104.

(26) Bauch, S.; Sørensen, L. K.; Madsen, L. B. Phys. Rev. A 2014, 90, 062508.

(27) Sonk, J. A.; Caricato, M.; Schlegel, H. B. J. Phys. Chem. A 2011, 115, 4678-4690.

(28) Sonk, J. A.; Schlegel, H. B. J. Phys. Chem. A 2011, 115, 11832-11840.

(29) Mignolet, B.; Gijsbertsen, A.; Vrakking, M. J. J.; Levine, R. D.; Remacle, F. Phys. Chem. Chem. Phys. 2011, 13, 8331-8344.

(30) Mignolet, B.; Johansson, J.; Campbell, E.; Remacle, F. ChemPhysChem 2013, 14, $3332-3340$.

(31) Kelkensberg, F.; Siu, W.; Pérez-Torres, J. F.; Morales, F.; Gademann, G.; Rouzée, A.; Johnsson, P.; Lucchini, M.; Calegari, F.; Sanz-Vicario, J. L.; Martín, F.; Vrakking, M. J. J. Phys. Rev. Lett. 2011, 10\%, 043002.

(32) Carpeggiani, P. A.; Tzallas, P.; Palacios, A.; Gray, D.; Martín, F.; Charalambidis, D. Phys. Rev. A 2014, 89, 023420.

(33) Lein, M. Phys. Rev. Lett. 2005, 94, 053004.

(34) Lorin, E.; Chelkowski, S.; Bandrauk, A. D. New J. Phys. 2008, 10, 025033. 


\section{Page 33 of 36}

(35) Fuks, J. I.; Luo, K.; Sandoval, E. D.; Maitra, N. T. 2014, arXiv:physics/1412.0586.

(36) Laurent, A. D.; Jacquemin, D. Int. J. Quant. Chem. 2013, 113, 2019-2039.

(37) Habenicht, B. F.; Tani, N. P.; Provorse, M. R.; Isborn, C. M. J. Chem. Phys. 2014, $141,184112$.

(38) Fuks, J. I.; Helbig, N.; Tokatly, I. V.; Rubio, A. Phys. Rev. B 2011, 84, 075107.

(39) Ruggenthaler, M.; Bauer, D. Phys. Rev. Lett. 2009, 102, 233001.

(40) Fuks, J. I.; Elliott, P.; Rubio, A.; Maitra, N. T. J. Phys. Chem. Lett. 2013, 4, 735-739.

(41) Fuks, J. I.; Maitra, N. T. Phys. Chem. Chem. Phys. 2014, 16, 14504-14513.

(42) Elliott, P.; Fuks, J. I.; Rubio, A.; Maitra, N. T. Phys. Rev. Lett. 2012, 109, 266404.

(43) Raghunathan, S.; Nest, M. J. Chem. Theory Comput. 2011, 7, 2492-2497.

(44) Raghunathan, S.; Nest, M. J. Chem. Theory Comput. 2012, 8, 806-809.

(45) Piecuch, P.; Kowalski, K.; Pimienta, I. S. O.; Fan, P. D.; Lodriguito, M.; McGuire, M. J.; Kucharski, S. A.; Kuś, T.; Musiał, M. Theor. Chem. Acc. 2004, 112, 349-393.

(46) Geertsen, J.; Rittby, M.; Bartlett, R. J. Chem. Phys. Lett. 1989, 164, 57-62.

(47) Krylov, A. I. Annu. Rev. Phys. Chem. 2008, 59, 433-462.

(48) Castro, A.; Appel, H.; Oliveira, M.; Rozzi, C. A.; Andrade, X.; Lorenzen, F.; Marques, M. A. L.; Gross, E. K. U.; Rubio, A. Phys. Stat. Sol. B 2006, 243, 2465-2488.

(49) Strubbe, D. A.; Lehtovaara, L.; Rubio, A.; Marques, M. A. L.; Louie, S. G. In Fundamentals of Time-Dependent Density Functional Theory; Marques, M. A. L., Maitra, N. T., Nogueira, F. M. S., Gross, E. K. U., Rubio, A., Eds.; Lecture Notes in Physics; Springer Verlag: Berlin, 2012; Vol. 837; pp 139-166. 
(50) Chelkowski, S.; Bandrauk, A. D. Phys. Rev. A 2002, 65, 061802.

(51) Andrade, X.; Alberdi-Rodriguez, J.; Strubbe, D. A.; Oliveira, M. J. T.; Nogueira, F.; Castro, A.; Muguerza, J.; Arruabarrena, A.; Louie, S. G.; Aspuru-Guzik, A.; Rubio, A.; Marques, M. A. L. J. Phys.: Condens. Matter 2012, 24, 233202.

(52) Hartwigsen, C.; Goedecker, S.; Hutter, J. Phys. Rev. B 1998, 58, 3641-3662.

(53) Castro, A.; Marques, M. A. L.; Rubio, A. J. Chem. Phys. 2004, 121, 3425-3433.

(54) Marques, M. A. L.; Oliveira, M. J. T.; Burnus, T. Comp. Phys. Comm. 2012, 183, $2272-2281$.

(55) Becke, A. D. J. Chem. Phys. 1993, 98, 5648-5652.

(56) Stephens, P. J.; Devlin, F. J.; Chabalowski, C. F.; Frisch, M. J. J. Phys. Chem. 1994, 98, 11623-11627.

(57) Legrand, C.; Suraud, E.; Reinhard, P. G. J. Phys. B: At. Mol. Opt. Phys. 2002, 35, $1115-1128$.

(58) Hessler, P.; Maitra, N. T.; Burke, K. J. Chem. Phys. 2002, 117, 72-81.

(59) Ullrich, C. A. J. Chem. Phys. 2006, 125, 234108.

(60) Muskatel, B. H.; Remacle, F.; Levine, R. D. Phys. Scr. 2009, 80, 048101.

(61) Werner, H.-J.; Knowles, P. J.; Knizia, G.; Manby, F. R.; Schütz, M. Comput. Mol. Sci. 2012, 2, 242-253.

(62) Remacle, F.; Levine, R. D. Phys. Rev. A 2011, 83, 013411.

(63) Mignolet, B.; Levine, R. D.; Remacle, F. Phys. Rev. A 2014, 89, 021403.

(64) Veryazov, V.; Malmqvist, P. .; Roos, B. O. Int. J. Quant. Chem. 2011, 111, 3329-3338. 


\section{Page 35 of 36}

(65) Mignolet, B.; Levine, R. D.; Remacle, F. J. Phys. Chem. A 2014, 118, 6721-6729.

(66) Stwalley, W. C.; Zemke, W. T. J. Phys. Chem. Ref. Data 1993, 22, 87-112.

(67) Wharton, L.; Gold, L. P.; Klemperer, W. J. Chem. Phys. 1962, 37, 2149-2150.

(68) Weber, A. M.; Acharya, A.; Parker, D. H. J. Phys. Chem. 1984, 88, 6087-6089. 